\title{
Correspondence
}

\section{Activity and specificity of necrostatin-1, small-molecule inhibitor of RIP1 kinase}

\author{
Cell Death and Differentiation (2013) 20, 366; doi:10.1038/cdd.2012.133; published online 30 November 2012
}

\section{Dear Editor,}

Necroptosis has emerged as an important form of regulated necrotic cell death, which can occur during mouse development and is also an important contributor to necrotic tissue injury in a variety of mouse models of human pathologies, including brain trauma, ischemia-reperfusion injuries, viral infections and acute inflammatory responses. ${ }^{1}$ Necrostatin-1 (Nec-1; methyl-thiohydantoin-tryptophan (MTH-Trp)), identified by us in a cell-based small-molecule necroptosis screen, ${ }^{2}$ was found to selectively target the kinase activity of RIP1, a key mediator of necroptosis. ${ }^{3} \mathrm{Nec}-1$ is commercially available and has been used extensively both in vitro and in vivo by multiple groups to elucidate the role of necroptosis. However, because this molecule is also known to inhibit indoleamine2,3-dioxygenase (IDO), ${ }^{4}$ we feel that it is important to revisit and summarize some of the key published and new data regarding activity and specificity of $\mathrm{Nec}-1$.

We assessed inhibition of RIP1 and IDO by Necs, including $\mathrm{Nec}-1$, original molecule identified in our screen, ${ }^{2}$ 7-Cl-O$\mathrm{Nec}-1$, optimized analog of Nec-1, 3,5 inactive analogs of both Necs (denoted by ' $l$ ') and an IDO inhibitor, 1-methyl-D, L-tryptophan (1-MT) (Supplemantary Figure S1A). With respect to RIP1 inhibition, our data confirmed excellent correlation of Nec's activity against RIP1 kinase in vitro and necroptosis in Jurkat cells (Supplemantary Figure S1B). Notably, 1-MT failed to inhibit necroptosis, confirming the specific role of RIP1 kinase, rather than IDO, in necroptosis. Conversely, only the original Nec-1, but none of the other Necs, inhibited IDO activity (Supplemantary Figure S1C). These results clearly distinguish the SAR of Necs against the two different enzyme targets.

As we pointed out in our papers, widely used and commercially available Necrostain-1 (MTH-Trp), a molecule originally identified in a necroptosis screen, is not optimal. ${ }^{2,3,5}$ In particular, metabolic stability of Nec-1 is very limited $\left(T_{1 / 2}<5\right.$ min in mouse microsomal assay) because of the presence of sulfur of thiohydantoin. ${ }^{5}$ Chemical optimization of Nec-1 (> 200 derivatives analyzed to date) led to the development of a much improved analog, termed 7-Cl-O-Nec-1 (or 7-Cl-O-MH-Trp) (Supplemantary Figure $\mathrm{S} 1 \mathrm{~A}),{ }^{5}$ which showed a robust improvement in metabolic stability to $\mathrm{T}_{1 / 2}$ of $\sim 1 \mathrm{~h}$ in liver microsomal assay and in in vivo pharmacokinetics (PK) study. ${ }^{5}$
Target specificity is a critical consideration for any smallmolecule tool. Notably, optimized 7-Cl-O-Nec-1 displayed exclusive selectivity towards RIP1 in a screen of a $>400$ human kinases, including several other RIP family members. ${ }^{6}$ Furthermore, we observed perfect correlation between inhibition of RIP1 kinase and cellular necroptosis by Nec-1 analogs. ${ }^{3}$ We also found, using wild-type and RIP1-deficient Jurkat and mouse fibroblast cells, that the ability of Nec-1 to inhibit cell death is entirely dependent on the expression of RIP1 kinase. ${ }^{3,6}$ Thus, Nec-1 and, especially, 7-Cl-O-Nec-1 represent useful chemical probes for defining the role of RIP1 kinase in cellular regulation. It is also worth noting that very closely related inactive analogs of both $\mathrm{Nec}-1$ and 7-Cl-O$\mathrm{Nec}-1$ have also been described to facilitate confirmation of on-target activity of the inhibitors in vitro and in vivo (Supplemantary Figures S1A and B). ${ }^{3}$

Overall, these data lead us to suggest that in interpreting the results obtained using original $\mathrm{Nec}-1$, a limited bioavailability and inhibitory activity against IDO should always be considered. It is also useful to bear in mind that Nec- $1 \mathrm{i}$, commercially available inactive derivative of $\mathrm{Nec}-1$, cannot be used alone to differentiate between targeting IDO and RIP1. Optimized 7-ClO-Nec-1 represents a superior tool, especially, in vivo. Additional tools, such as described in the literature RIP3 and IDOdeficient animals and/or short hairpin RNA knockdowns, can help further distinguish between these two pathways.

\section{Conflict of Interest}

The authors declare no conflict of interest.

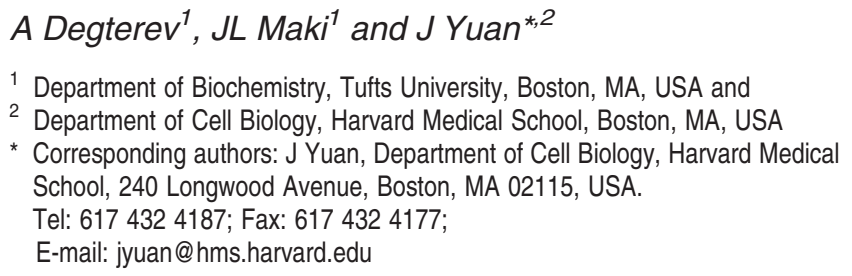

1 Department of Biochemistry, Tufts University, Boston, MA, USA and 2 Department of Cell Biology, Harvard Medical School, Boston, MA, USA

* Corresponding authors: J Yuan, Department of Cell Biology, Harvard Medical School, 240 Longwood Avenue, Boston, MA 02115, USA.

Tel: 617432 4187; Fax: 617432 4177;

E-mail: jyuan@ $\mathrm{hms}$.harvard.edu

\footnotetext{
1. Galluzzi L et al. Int Rev Cell Mol Biol 2011; 289: 1-35.

2. Degterev A et al. Nat Chem Biol 2005; 1: 112-119.

3. Degterev A et al. Nat Chem Biol 2008; 4: 313-321.

4. Muller AJ et al. Nat Med 2005; 11: 312-319.

5. Teng X et al. Bioorg Med Chem Lett 2005; 15: 5039-5044.

6. Christofferson DE et al. Cell Death Dis 2012; 3: e320.
} 